\title{
II-6 is non-essential to murine CD19 CAR efficacy, but can mediate acute GVHD following allogeneic BMT with CAR T cell infusion
}

\author{
Elad Jacoby ${ }^{1 *}$, Haiying Qin², James Kochenderfer ${ }^{3}$, Yinmeng Yang ${ }^{1}$, Chris Chien ${ }^{1}$, Terry Fry ${ }^{4}$ \\ From 30th Annual Meeting and Associated Programs of the Society for Immunotherapy of Cancer \\ (SITC 2015) \\ National Harbor, MD, USA. 4-8 November 2015
}

Chimeric-antigen-receptor (CAR) T cells targeting CD19 show dramatic remissions in refractory or relapsed acute lymphoblastic leukemia. Interleukin-6 (IL-6) has been associated with severe cytokine release syndrome (CRS) following CAR $\mathrm{T}$ cell treatment, leading to significant toxicity and death. CRS could be treated with an antiIL6-receptor antibody, with reversal of most of the inflammatory response within hours to days. In addition, the significance of IL- 6 in prevention and treatment of graft-versus-host disease (GVHD) following allogeneic bone marrow transplant (allo-BMT) has been shown in pre-clinical and clinical settings.

Here, we used an immunocompetent murine model of ALL to study CD19 CAR biology. In a syngeneic/ autologous model, we found no significant increase in IL-6 in leukemia bearing mice following CD19 CAR treatment. No significant alteration in efficacy was seen when administering CD19 CAR from wild-type mice or IL6-/mice to wild-type or IL6-/-recipients, with all achieving long term remissions compared to controls.

To elucidate IL-6 in this system, we used a minor-mismatch allo-BMT mouse model (B6àC3h.sw), previously shown to have increased IL-6 levels when T cell replete BMT given. Using a T cell depleted allo-BMT platform, we saw significant increase in IL-6 in leukemia bearing mice treated with CD19 CAR T cells compared to leukemia-bearing controls treated with mock-T cells, and non-leukemia bearing allogeneic CAR recipients. Peak of IL-6 levels was seen 5 days following CAR-T cell infusion, either when given in proximity to the BMT or in later time points. The rise in IL-6 correlated with an acute systemic inflammatory process resembling acute GVHD, that caused early lethality despite being a minor-mismatch model, and at relatively low $\mathrm{T}$ cell doses. This acute GVHD could not be reversed when $\mathrm{T}$ cells were given early (day 0-2 post BMT), using either IL6-receptor blockade or IL6-/-bone marrow on day 0 . However, when leukemia and CD19 CAR administered late $($ day $+12-+17)$ following allo-BMT, wild-type marrow recipients still died of acute GVHD while IL6-/-recipients were rescued.

Altogether, we show that IL-6 is not essential for CD19 CAR efficacy in this murine model, but can drive significant toxicity following an allogeneic BMT with CAR.

\section{Authors' details}

${ }^{1}$ Pediatric Oncology Branch, National Cancer Institute, Bethesda, MD, USA. ${ }^{2} \mathrm{NCl}, \mathrm{NIH}$, Bethesda, MD, USA. ${ }^{3}$ National Cancer Institute, Bethesda, MD, USA. ${ }^{4}$ Pediatric Oncology Branch, CCR, NCl, NIH, Bethesda, MD, USA.

Published: 4 November 2015

doi:10.1186/2051-1426-3-S2-P226

Cite this article as: Jacoby et al:: II-6 is non-essential to murine CD19 CAR efficacy, but can mediate acute GVHD following allogeneic BMT with CAR T cell infusion. Journal for ImmunoTherapy of Cancer 2015 3(Suppl 2):P226. 